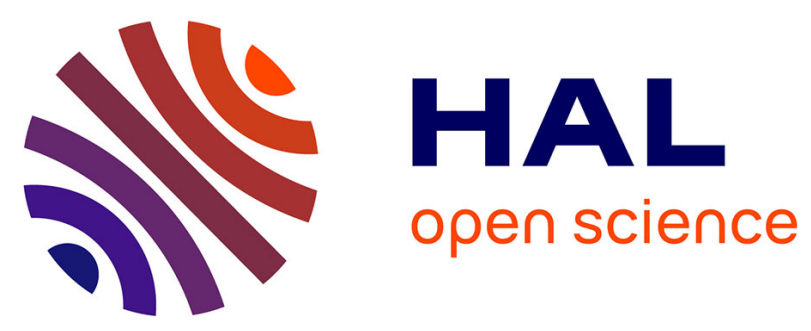

\title{
Etude de l'hétérosis chez le colza oléagineux d'hiver (Brassica napus L.) I. - Comparaison de deux populations, l'une homozygote et l'autre hétérozygote \\ Marianne Lefort-Buson, Yvette Dattée
}

\section{- To cite this version:}

Marianne Lefort-Buson, Yvette Dattée. Etude de l'hétérosis chez le colza oléagineux d'hiver (Brassica napus L.) I. - Comparaison de deux populations, l'une homozygote et l'autre hétérozygote. Agronomie, 1985, 5 (2), pp.101-110. hal-00884738

\section{HAL Id: hal-00884738 \\ https://hal.science/hal-00884738}

Submitted on 1 Jan 1985

HAL is a multi-disciplinary open access archive for the deposit and dissemination of scientific research documents, whether they are published or not. The documents may come from teaching and research institutions in France or abroad, or from public or private research centers.
L'archive ouverte pluridisciplinaire HAL, est destinée au dépôt et à la diffusion de documents scientifiques de niveau recherche, publiés ou non, émanant des établissements d'enseignement et de recherche français ou étrangers, des laboratoires publics ou privés. 


\title{
Etude de l'hétérosis chez le colza oléagineux d'hiver (Brassica napus L.) I. - Comparaison de deux populations, l'une homozygote et l'autre hétérozygote
}

\author{
Marianne LEFORT-BUSON \& Yvette DATTÉE $\left({ }^{*}\right)$ \\ I.N.R.A., Station d'Amélioration des Plantes, Centre de Recherches de Rennes, B.P. 29, F 35650 Le Rheu \\ (*) I.N.R.A., Université de Paris XI, Laboratọire d'Amélioration des Plantes, F 91405 Orsay Cedex
}

RÉSUMÉ

\begin{abstract}
L'article présente une synthèse de 2 années d'études sur la comparaison d'une population de lignées d'origines diverses et d'une population d'hybrides F1 issus de ces lignées. Des échantillons de chaque population, différents les 2 années d'essai, ont été testés au champ en petites parcelles à la densité de $4 \mathrm{~kg} / \mathrm{ha}$.

Les populations homozygotes et hétérozygotes ont été comparées ; l'étude a porté sur la distribution de différents caractères agronomiques dans chaque population et sur les corrélations entre leurs résidus. A l'exception de la valeur moyenne de la population souvent plus élevée chez les hybrides, on observe une grande similitude de comportement entre les homozygotes et les hétérozygotes. Ces résultats apportent les premiers éléments d'une discussion sur la nature de l'hétérosis chez le colza.

Par ailleurs, l'amplitude de la vigueur hybride moyenne, bien que variable selon l'année, souligne l'intérêt des
\end{abstract} variétés hybrides $\mathrm{F} 1$ pour l'amélioration de la productivité du colza oléagineux.

Mots clés additionnels : Vigueur hybride, corrélations environnementales. populations.

\begin{abstract}
The paper presents the results of a two-year comparison of two populations : one with different inbred lines and the other with F1 hybrids originating from these lines. Different samples were taken from each population in each experimental year and tested in small field plots at a sowing rate of $4 \mathrm{~kg} / \mathrm{ha}$. Homozygous and heterozygous populations were compared. The distribution of several agronomic characters in each population was studied, and the correlations between the residual variables. Apart from the population mean, which was often greater in the Fl hybrids, most estimates were similar for the homozygous and heterozygous populations. The results provide a preliminary basis for discussing the nature of heterosis in oilseed rape. Average hybrid vigor was estimated : it depended on the year, but nevertheless confirmed the value of producing $F 1$ hybrid varieties in order to improve rapeseed productivity.
\end{abstract}

Additional key words : Hybrid vigor, environmental correlations.

\section{INTRODUCTION}

Le système de reproduction mixte $(2 / 3$ autofécondation, 1/3 allofécondation) du colza (Brassica napus L.) a conduit à la sélection de 2 types variétaux; les lignées considérées comme homozygotes et les variétés «populations » partiellement hétérozygotes. Les premières sont issues de nombreux cycles d'autofécondation contrôlée à partir de populations ou de croisements, les secondes de fécondations libres entre géniteurs sélectionnés.
La découverte de systèmes de stérilité mâle génocytoplasmiques chez B. napus (THOMPSON, 1972; SHIGA, 1976) et les possibilités d'utilisation de ceux-ci en sélection (ROUSSELlE, 1982; ROUSSELLE \& RENARD, 1982) permettent d'envisager la création d'hybrides F1. Ces variétés apporteraient un gain sensible de productivité et de vigueur par rapport aux lignées et aux "populations " (SCHUSTER, 1969; SHELKUDENKO, 1968, 1972 ; SCHUSTER \& MICHAEL, 1976 ; CAMPBEL \& KONDRA, 1978 ; LEFORT-BUSON \& DATTÉE, 1982). 
Afin de contribuer à la compréhension et à l'exploitation de l'hétérosis chez le colza oléagineux, nous présentons ici une synthèse de 2 années d'étude sur un échantillonnage important d'hybrides F1 et de leurs lignées parentales. Ce $1^{\text {er }}$ article est consacré à la comparaison des populations variétales hétérozygotes et homozygotes et à l'estimation de la vigueur hybride moyenne de la population de lignées.

Les composantes génétiques de la variance de la population de lignées et les orientations à donner pour la sélection d'hybrides $F 1$ seront étudiées dans un $2^{\mathrm{e}}$ article.

\section{MATÉRIEL ET MÉTHODES}

\section{A. Matériel végétal ; plan de croisement ; dispositif expérimental}

L'étude porte sur la population de lignées sélectionnées représentée par la collection de géniteurs de la Station d'Amélioration des Plantes de Rennes (I.N.R.A.). En 1978, 35 lignées d'origines géographiques et/ou génétiques variées, issues de la population sélectionnée, ont été croisées manuellement 5 par 5 selon 7 diallèles élémentaires : 140 hybrides directs et réciproques et leurs lignées parentales ont été testés au champ pendant la campagne 1978/79. En 1980, un autre échantillon de la population a été utilisé pour confirmer les résultats de 1979 : 30 lignées ont été croisées 5 par 5 selon 6 diallèles (sans différencier les effets réciproques) ; 60 hybrides F1 ont été produits et comparés aux lignées parentales en 1980/81. Pour bâtir le plan de croisement, les lignées ont été réparties respectivement dans les $7(1978)$ et $6(1980)$ diallè- les élémentaires, en limitant au maximum les possibilités d'apparentement génétique et/ou géographique à l'intérieur d'un dispositif élémentaire (BUSON, 1979). Différents témoins ont été utilisés :

- la lignée "Primor » a servi de géniteur dans tous les diallèles en 1979,

- la lignée "Kid » a été placée dans tous les blocs expérimentaux en 1981.

Les dispositifs expérimentaux incluant parents et hybrides étaient respectivement en 1979 et 1981 des blocs quasi-complets à 2 et 4 répétitions, avec des parcelles de 4 et 5 lignes de $3 \mathrm{~m}$. La densité de semis était d'environ $4 \mathrm{~kg} / \mathrm{ha}$.

\section{B. Caractères mesurés}

En 1979, 18 caractères ont été mesurés : 10 mesures étaient parcellaires et 8 ont été effectuées sur des échantillons de plantes. Cependant, les échantillons prélevés étaient trop petits pour obtenir une précision suffisante (LEFORT-BUSON \& DATTÉE, 1982) et l'augmentation de leur taille était matériellement impossible. L'étude a donc été restreinte à 7 caractères en 1981.

A l'exception de la sensibilité à la verse qui n'a pu être notée en 1981 du fait des conditions climatiques, nous ne présentons ici que les résultats relatifs aux caractères pouvant être comparés sur les 2 années (tabl. 1). Pour certains caractères, les méthodes de mesure ou d'échantillonnage ont été modifiées en 1981 afin de gagner en précision. Par ailleurs, le poids de grains par silique a été introduit en 1981 pour rechercher si ce caractère pouvait être substitué au nombre de grains par silique dans un but de simplification expérimentale.

TABLEAU 1

Description des caractères et de la méthode d'échantillonnage.

Description of the characters and the sampling method.

Caractère mesuré

1979
Echelle de notation, unité de mesure

Méthode d'échantillonnage

1981

Date de début floraison (DF) Nombre de jours séparant la date de début floraison de la variété considérée et de la variété la plus précoce

\begin{tabular}{lcc}
\hline $\begin{array}{l}\text { Couvert foliaire au début } \\
\text { floraison (CF) }\end{array}$ & $\begin{array}{c}0,5 \text { (peu de végétation) } \\
\text { à } 8 \text { (parcelle très fournie) }\end{array}$ & 0,5 à 6 \\
\hline Rendement parcellaire (RENDT) & $\begin{array}{c}\text { Poids de grains secs sur les lignes centrales de la parcelle (en q/ha), la récolte des seules lignes } \\
\text { centrales permettant de limiter la compétition intervariétale (LEFORT-Buson, 1981) }\end{array}$ \\
\hline $\begin{array}{l}\text { Poids de } 1000 \text { grains (P 1000) } \\
\text { Estimé à partir d'un échantillon } \\
\text { de } 8 \text { plantes (en g) }\end{array}$ & $\begin{array}{c}\text { Estimé à partir de } 3 \text { échantillons parcellaires } \\
\text { de } 1000 \text { grains (en g) }\end{array}$ \\
\hline $\begin{array}{l}0 \text { (absence de verse) } \\
\text { ànsibilité à la verse (VERS) }\end{array}$ & (parcelle très versée) & Non mesuré
\end{tabular}

Nombre de grains par silique (NG/SIL)
Estimé sur les ramifications primaires (NG/SIL. RI) et secondaires (NG/SIL. RII) à partir d'un échantillon de 8 plantes
Estimé à partir de 2 échantillons parcellaires de 50 siliques (NG/SIL)

Poids de grains par silique

(PG/SIL)

Non mesure

Estimé à partir des échantillons utilisés pour la mesure du nombre de grains par silique (en $\mathrm{mg}$ )

NB : Les sigles entre parenthèses seront utilisés pour les tableaux ultérieurs

NB : The symbols in brackets are used in the other tables. 


\section{Méthode d'étude}

Pour chaque population homozygote et hétérozygote et pour chaque diallèle élémentaire, les résultats ont été traités selon un modèle d'analyse de variance à 2 facteurs (famille et bloc). L'analyse a été poursuivie par:

- l'étude des variances et des covariances résiduelles intrapopulations ;

- l'étude des distributions des caractères dans chaque population.

Du fait de la nature génétique des 2 populations, la variance résiduelle est supposée correspondre à la variance environnementale. L'homogénéité des variances résiduelles des diallèles élémentaires a été éprouvée par un test de Bartlett à 5 p. 100, indépendamment chez les lignées et chez les hybrides F1; le test a été effectué pour chaque année d'expérimentation. Dans un second temps, pour chaque caractère, les variances résiduelles des populations homozygotes et hétérozygotes ont été comparées selon un test de Fisher à 1 p. 100, soit avec un risque global de 7 et 6 p. 100 respectivement en 1979 et 1981 pour l'ensemble des caractères. Enfin, les corrélations entre résidus ont été étudiées dans chaque population. Ces corrélations traduisent la liaison entre les effets non directement génétiques et pourraient constituer une caractéristique des populations en interaction avec le milieu. La comparaison des matrices de corrélations associées aux 2 populations n'a pu être effectuée, faute de test satisfaisant. Toutefois, du fait de l'égalité des variances résiduelles dans les 2 populations, les matrices de covariances ont été comparées selon une méthode proposée par BARTLETT (in ANDERSON, 1957).

Les histogrammes relatifs aux moyennes de chaque caractère ont été dressés à titre indicatif pour les 2 populations après avoir centré chaque estimation par rapport à celle du témoin de chaque dispositif élémentaire. Cependant, l'ajustement à des distributions normales n'a pas été calculé du fait du trop faible nombre de variétés.

Les variances des moyennes variétales ont été estimées dans les 2 populations et comparées par un test de Fisher à 1 p. 100 pour chaque caractère (soit avec un risque de 7 à 6 p. 100 respectivement en 1979 et 1981 pour l'ensemble des caractères). Les variances génétiques ont aussi été estimées.

La comparaison de la moyenne des performances des hybrides à celle des lignées (test de Student à 5 p. 100 , bilatéral) permet de rechercher l'importance de la vigueur hybride moyenne dans la population.

Dans toute la suite de l'article, les résultats de 1979 et 1981 sont présentés en parallèle pour permettre de juger de leur homogénéité ; c'est pourquoi sont mentionnées à nouveau quelques données de l'essai de 1979 sur la vigueur hybride et les corrélations entre résidus (LEFORT-BUSON \& DATTÉE, 1982). Néanmoins, les échantillons variétaux testés en 1979 et 1981 étaient trop différents pour que puisse être effectuée une comparaison globale des résultats des 2 années.

\section{RÉSULTATS}

\section{A. Variances et covariances résiduelles intrapopula- tions}

\section{Homogénéité des diallèles élémentaires}

Les résultats individuels sont présentés dans les annexes I et II. Dans les 2 populations, le rapport de la plus grande à la plus petite variance varie le plus fréquemment, de 1,3 à 2 avec une valeur maximum égale à 3,1 (cas du rendement chez les hybrides en 1979). L'hypothèse d'homogénéité des variances des dispositifs élémentaires est acceptée pour les 2 populations et les 2 années (tabl. 2). Ce résultat constitue un

TABLEAU 2

Variances résiduelles : estimations globales ponctuelles et par intervalle dans les essais de 1979 (a) et 1981 (b). Overall estimations with confidence limits of residual variances for two trials, 1979 (a) and 1981 (b).

\begin{tabular}{|c|c|c|c|c|c|}
\hline Caractère & & $\chi^{2}(\mathrm{c})$ & $\begin{array}{c}\text { Hybrides } \\
\hat{\gamma} \mathrm{e}\end{array}$ & $\chi^{2}(\mathrm{c})$ & $\begin{array}{l}\text { Lignées } \\
\hat{\gamma} e\end{array}$ \\
\hline DF & $\begin{array}{l}\text { (a) } \\
\text { (b) }\end{array}$ & $\begin{array}{l}2,023 \\
7,016\end{array}$ & $\begin{array}{l}1,070<1,406<1,782 \\
0,851<1,051<1,295\end{array}$ & $\begin{array}{l}1,718 \\
5,144\end{array}$ & $\begin{array}{l}1,228<1,718<2,574 \\
1,262<1,685<2,364\end{array}$ \\
\hline $\mathrm{CF}$ & $\begin{array}{l}\text { (a) } \\
\text { (b) }\end{array}$ & $\begin{array}{l}1,924 \\
0,534\end{array}$ & $\begin{array}{l}0,728<0,956<1,211 \\
0,212<0,261<0,322\end{array}$ & $\begin{array}{l}1,244 \\
0,917\end{array}$ & $\begin{array}{l}0,844<1,181<1,769 \\
0,217<0,290<0,407\end{array}$ \\
\hline RENDT & $\begin{array}{l}\text { (a) } \\
\text { (b) }\end{array}$ & $\begin{array}{l}9,857 \\
1,930\end{array}$ & $\begin{array}{l}19,43<25,53<32,37 \\
21,80<26,89<32,13\end{array}$ & $\begin{array}{l}1,214 \\
0,400\end{array}$ & $\begin{array}{l}20,76<29,05<43,53 \\
16,13<21,54<30,22\end{array}$ \\
\hline P 1000 & $\begin{array}{l}\text { (a) } \\
\text { (b) }\end{array}$ & $\begin{array}{l}2,322 \\
4,308\end{array}$ & $\begin{array}{l}0,1399<0,1838<0,2330 \\
0,0335<0,0413<0,0509\end{array}$ & $\begin{array}{l}2,769 \\
2,404\end{array}$ & $\begin{array}{l}0,1589<0,2223<0,3331 \\
0,0366<0,0489<0,0686\end{array}$ \\
\hline VERSE & $\begin{array}{l}\text { (a) } \\
\text { (b) }\end{array}$ & $\begin{array}{c}7,471 \\
-\end{array}$ & $\begin{array}{c}0,450<0,591<0,749 \\
-\end{array}$ & $\begin{array}{c}1,444 \\
-\end{array}$ & $0,465<0,651<0,975$ \\
\hline NG/SIL.I & $\begin{array}{l}\text { (a) } \\
\text { (b) }\end{array}$ & $\begin{array}{c}6,296 \\
-\end{array}$ & $\begin{array}{c}5,853<7,689<9,748 \\
-\end{array}$ & $\begin{array}{c}1,879 \\
-\end{array}$ & $\begin{array}{c}4,523<6,328<9,482 \\
-\end{array}$ \\
\hline NG/SIL.II & $\begin{array}{l}\text { (a) } \\
\text { (b) }\end{array}$ & $\begin{array}{c}6,069 \\
-\end{array}$ & $\begin{array}{c}3,431<4,508<5,175 \\
-\end{array}$ & $\begin{array}{c}2,093 \\
-\end{array}$ & $\begin{array}{c}3,368<4,711<7,059 \\
-\end{array}$ \\
\hline NG/SIL & $\begin{array}{l}\text { (a) } \\
\text { (b) }\end{array}$ & $\overline{1,204}$ & $4,802<5, \overline{924}<7,298$ & $\overline{0,319}$ & $5,261<7, \overline{026}<9,859$ \\
\hline $\mathrm{PG} / \mathrm{SIL}$ & $\begin{array}{l}\text { (a) } \\
\text { (b) }\end{array}$ & $\overline{0,673}$ & $108,9<134,4<165,6$ & $\overline{1,104}$ & $111,6<149,1<209,2$ \\
\hline
\end{tabular}

(c) Les valeurs seuil du $\chi^{2}$ à $6(1979)$ et $5(1981)$ ddl, avec un risque de $5 \%$, sont respectivement de 12,59 et 11,07 .

(c) Threshold values for a $\chi^{2}$ with 6 (1979) and 5 (1981) df, and with a $5 \%$ risk are 12.59 and 11.07 respectively. 
argument en faveur de l'appartenance des lignées des différents diallèles élémentaires à une même population ; la même conclusion est admise pour les hybrides. Dans la suite des analyses, la comparaison portera donc sur les 2 populations : lignées et hybrides.

\section{Comparaison des 2 années et des 2 populations}

A l'exception des caractères " couvert foliaire " et "poids de 1000 grains " pour lesquels la valeur de 1979 est 3 à 4 fois plus grande que celle de 1981 (tabl. 2), les variances résiduelles sont du même ordre de grandeur les 2 années, chez les hybrides comme chez les lignées. Pour le poids de 1000 grains, la différence peut provenir du mode d'échantillonnage : un prélèvement parcellaire global (1981) peut être plus précis que l'analyse d'un nombre restreint de plantes (1979). En revanche, pour le couvert foliaire, la différence entre les 2 échelles de notation ne semble pas pouvoir à elle seule expliquer l'hétérogénéité des variances.

Quel que soit le caractère, les valeurs des variables de Fisher calculées pour comparer les variances des lignées à celle des hybrides ne dépassent pas 1,2 et 1,6 respectivement en 1979 et en 1981. L'hypothèse d'homogénéité des variances résiduelles des 2 populations est donc acceptée globalement pour l'ensemble des caractères. Cependant, pour 6 caractères sur 7 en 1979 et 5 caractères sur 6 en 1981, la variance associée aux lignées est supérieure à celle associée aux hybrides ; cette tendance pourrait traduire une meilleure homéostase des hybrides.

\section{Corrélations et covariances résiduelles}

Les estimées des corrélations entre résidus sont faibles à l'exception de celle qui lie les nombres et poids de grains par silique (tabl. 3). Dans ce dernier cas, la valeur élevée de la corrélation $(\hat{\rho} \# 0,9)$ suggère de se limiter ultérieurement à l'étude du poids moyen de grains par silique ; cette liaison a d'ailleurs été confirmée dans d'autres expériences à la fois entre les résidus et entre les valeurs phénotypiques (LEFORTBuson, non publié).

Mises à part les estimations voisines de 0 et par conséquent non significatives, on remarque une grande similitude entre les corrélations obtenues en 1979 et 1981.

Quelle que soit la population ou l'année (tabl. 3) :

- la date de début floraison et le couvert foliaire sont en corrélation négative $(\hat{\rho} \#-0,2)$ et significative dans 3 cas sur 4 ;

- le rendement et le nombre de grains par silique sont en corrélation positive ( $\hat{\rho} \# 0,2)$ et significative dans 3 cas sur 4 si on prend en compte les estimations obtenues en 1979 à partir des ramifications secondaires. En revanche, la corrélation entre le rendement et le nombre de grains par silique de la ramification principale (estimé en 1979) est faible. Il a été montré que les ramifications secondaires expliquent plus de 85 p. 100 du rendement global d'une plante (LEFORTBUSON, non publié) ; la corrélation calculée en 1981 entre le rendement et le nombre de grains par silique mesuré globalement sur l'ensemble des ramifications traduit donc certainement la liaison avec la production des ramifications secondaires (tabl. 3);

\section{TABLEAU 3}

Estimation des corrélations résiduelles dans les populations homozygote (en dessous de la diagonale) et hétérozygote (au-dessus de la diagonale), dans les essais de 1979 (a) et 1981 (b).

Estimates of the residual correlations in homozygous (below the diagonal) and heterozygous (above the diagonal) populations for the two trials, 1979 (a) and 1981 (b).

\begin{tabular}{|c|c|c|c|c|c|c|c|c|c|c|}
\hline \multirow[b]{2}{*}{ Homozygotes } & \multicolumn{3}{|c|}{ Hétérozygotes } & \multirow[b]{2}{*}{ RENDT } & \multirow[b]{2}{*}{ P 1000} & \multirow[b]{2}{*}{ VERSE } & \multirow[b]{2}{*}{ NG/SIL.I } & \multirow[b]{2}{*}{ NG/SIL.II } & \multirow[b]{2}{*}{ NG/SIL } & \multirow[b]{2}{*}{ PG/SIL } \\
\hline & & $\mathrm{DF}$ & $\mathrm{CF}$ & & & & & & & \\
\hline DF & $\begin{array}{l}\text { (a) } \\
\text { (b) }\end{array}$ & & $\begin{array}{l}-0,255^{*} \\
-0,195^{*}\end{array}$ & $\begin{array}{r}0,110 \\
0,063\end{array}$ & $\begin{array}{l}-0,058 \\
-0,070\end{array}$ & $\begin{array}{l}0,093 \\
-\end{array}$ & $\begin{array}{c}-0,045 \\
-\end{array}$ & $\begin{array}{c}-0,086 \\
-\end{array}$ & $-\overline{0,104}$ & $-\overline{0,030}$ \\
\hline $\mathrm{CF}$ & $\begin{array}{l}\text { (a) } \\
\text { (b) }\end{array}$ & $\begin{array}{l}-0,207 \\
-0,236^{*}\end{array}$ & & $\begin{array}{l}0,147 \\
0,177\end{array}$ & $\begin{array}{r}0,039 \\
-0,046\end{array}$ & $\begin{array}{l}0,063 \\
-\end{array}$ & $\begin{array}{l}0,052 \\
-\end{array}$ & $\begin{array}{l}0,184 \\
-\end{array}$ & $-\overline{0,033}$ & $\overline{0,068}$ \\
\hline RENDT & $\begin{array}{l}\text { (a) } \\
\text { (b) }\end{array}$ & $\begin{array}{r}0,096 \\
0,028\end{array}$ & $\begin{array}{l}0,136 \\
0,119\end{array}$ & & $\begin{array}{l}0,132 \\
0,158\end{array}$ & $\begin{array}{l}0,035 \\
-\end{array}$ & $\begin{array}{l}0,075 \\
-\end{array}$ & $\begin{array}{l}0,431^{*} \\
-\end{array}$ & $\overline{0,200}$ & $\overline{0,191}$ \\
\hline P 1000 & $\begin{array}{l}\text { (a) } \\
\text { (b) }\end{array}$ & $\begin{array}{r}0,055 \\
-0,038\end{array}$ & $\begin{array}{r}0,033 \\
-0,029\end{array}$ & $\begin{array}{l}0,099 \\
0,076\end{array}$ & & $\begin{array}{c}-0,021 \\
-\end{array}$ & $-0,165$ & $-0,424^{*}$ & $-\overline{0,037}$ & $\overline{0,131}$ \\
\hline VERSE & $\begin{array}{l}\text { (a) } \\
\text { (b) }\end{array}$ & $\begin{array}{c}-0,048 \\
-\end{array}$ & $\begin{array}{l}0,082 \\
-\end{array}$ & $\begin{array}{c}-0,021 \\
-\end{array}$ & $\begin{array}{l}0,008 \\
-\end{array}$ & & $\begin{array}{l}0,058 \\
-\end{array}$ & $\begin{array}{c}0,039 \\
-\end{array}$ & - & - \\
\hline NG/SIL.I & $\begin{array}{l}\text { (a) } \\
\text { (b) }\end{array}$ & $\begin{array}{c}-0,038 \\
-\end{array}$ & $\begin{array}{l}0,047 \\
-\end{array}$ & $\begin{array}{l}0,095 \\
-\end{array}$ & $\begin{array}{l}0,026 \\
-\end{array}$ & $\begin{array}{l}0,054 \\
-\end{array}$ & & $\begin{array}{l}0,307^{*} \\
-\end{array}$ & - & - \\
\hline NG/SIL.II & $\begin{array}{l}\text { (a) } \\
\text { (b) }\end{array}$ & $\begin{array}{c}-0,057 \\
-\end{array}$ & $\begin{array}{l}0,102 \\
-\end{array}$ & $\begin{array}{l}0,351^{*} \\
-\end{array}$ & $\begin{array}{c}-0,258^{*} \\
-\end{array}$ & $\begin{array}{l}0,028 \\
-\end{array}$ & $\begin{array}{l}0,512^{*} \\
-\end{array}$ & & - & - \\
\hline NG/SIL & $\begin{array}{l}\text { (a) } \\
\text { (b) }\end{array}$ & $\overline{0,049}$ & $\overline{0,086}$ & $\overline{0,220^{*}}$ & $\overline{0,191}$ & - & - & - & & $\overline{0,892 *}$ \\
\hline $\mathrm{PG} / \mathrm{SIL}$ & $\begin{array}{l}\text { (a) } \\
\text { (b) }\end{array}$ & $\overline{0,029}$ & $\overline{0,092}$ & $\overline{0,249^{*}}$ & $-\overline{0,019}$ & - & - & - & $\overline{0,904 *}$ & \\
\hline
\end{tabular}


- le rendement et le couvert foliaire sont liés positivement $(\hat{\rho} \# 0,15)$. La corrélation estimée globalement sur les lignées et les hybrides est significative en 1979 et 1981 , bien que les estimations individuelles ne le soient pas.

L'hypothèse $d$ 'homogénéité des corrélations entre les 2 populations, testée par un test de $\chi^{2}$ à 5 p. 100 dans chaque cas où 1 valeur au moins sur les 2 était significativement différente de 0 (soit pour 5 couples de caractères en 1979 et 6 en 1981), a toujours été acceptée.

De plus, l'hypothèse d'égalité des 2 matrices de covariances a aussi été acceptée avec un risque de 5 p. 100. Il semble donc y avoir une bonne concordance pour l'ensemble des caractères entre le comportement des lignées et des hybrides vis-à-vis des conditions environnementales.

\section{B. Distribution des caractères chez les lignées et les hybrides}

Pour chaque caractère, les différences entre les hybrides ou entre les lignées sont significatives les 2 années. Les effets réciproques, peu importants dans l'essai de 1979 (LEFORT-BUSON \& DATTÉE, 1982), ne sont pas pris en compte ici.

\section{Histogrammes. Caractéristiques des populations}

Une étude purement qualitative montre que la plupart des distributions se rapprochent de la normalité (voir annexes III et IV). On observe cependant une légère dissymétrie pour la date de début floraison quelles que soient la population et l'année et pour le couvert foliaire chez les lignées. Par ailleurs, l'écart entre les moyennes des 2 populations varie selon les caractères, mais il y a toujours une zone de recouvrement entre les 2 distributions. Il n'y a donc aucune discontinuité entre les populations de plantes homozygotes et hétérozygotes dans l'expression des différents caractères.

Les variances génétiques $(\hat{\gamma} g)$ ont été estimées (tabl. 4). Elles sont du même ordre de grandeur dans les 2 populations, à l'exception des caractères « date de début floraison » et «poids de 1000 grains » en 1981 , et « résistance à la verse » en 1979. Dans ces 3 cas, la variance génétique entre les lignées est nettement supérieure à celle des hybrides ; la comparaison ne peut pas être établie statistiquement car la loi des estimateurs des variances génétiques n'est pas une loi classique.

Dans les 2 populations, la variance génétique semble plus faible en 1981 qu'en 1979 pour le rendement et le poids de 1000 grains et plus élevée en 1981 pour la date de début floraison. Ce résultat peut s'expliquer d'abord par un effet de l'année et éventuellement par la non-identité des lignées échantillonnées en 1979 et 1981.

\section{Comparaison des moyennes. Estimation de la vigueur hybride}

La vigueur hybride calculée par rapport au parent moyen est significative les 2 années pour la date de début floraison, le couvert foliaire, le rendement en grains, le poids de 1000 grains, ainsi que pour les nombres et poids de grains par silique en 1981 et pour la sensibilité à la verse en 1979 (tabl. 5). Les manifestations de l'hétérosis sont plus importantes pour un caractère "global » comme le rendement en grains que pour chacune des composantes étudiées : nombre et poids moyen de grains par silique, poids de 1000 grains.

TABLEAU 4

Valeur moyenne de la population ( $\hat{\mu} g)$ et dispersion des effets variétaux $(\hat{\gamma} g)$ dans les essais de 1979 (a) et 1981 (b). Mean value of the population ( $\hat{\mu} g)$ and dispersion of varietal effects ( $\hat{\gamma} g)$ for the two trials, 1979 (a) and 1981 (b).

\begin{tabular}{|c|c|c|c|c|c|}
\hline \multirow[b]{2}{*}{ Caractère } & & \multicolumn{2}{|c|}{ Lignées } & \multicolumn{2}{|c|}{ Hybrides F1 } \\
\hline & & $\hat{\mu}$ & $\hat{\gamma} \mathrm{g}$ & $\hat{\mu}$ & $\hat{\gamma} \mathrm{g}$ \\
\hline DF & $\begin{array}{l}\text { (a) } \\
\text { (b) }\end{array}$ & $\begin{array}{l}11,37 \pm 0,31 \\
14,74 \pm 0,24\end{array}$ & $\begin{array}{r}9,57 \\
17,97\end{array}$ & $\begin{array}{l}10,60 \pm 0,16 \\
13,44 \pm 0,13\end{array}$ & $\begin{array}{l}6,83 \\
9,79\end{array}$ \\
\hline $\mathrm{CF}$ & $\begin{array}{l}\text { (a) } \\
\text { (b) }\end{array}$ & $\begin{array}{l}3,37 \pm 0,26 \\
3,20 \pm 0,10\end{array}$ & $\begin{array}{l}0,839 \\
0,199\end{array}$ & $\begin{array}{l}5,24 \pm 0,12 \\
3,99 \pm 0,07\end{array}$ & $\begin{array}{l}0,713 \\
0,092\end{array}$ \\
\hline RENDT & $\begin{array}{l}\text { (a) } \\
\text { (b) }\end{array}$ & $\begin{aligned} 31,7 & \pm 1,3 \\
15,63 & \pm 0,85\end{aligned}$ & $\begin{array}{l}8,58 \\
8,28\end{array}$ & $\begin{aligned} 38,9 & \pm 0,6 \\
22,31 & \pm 0,67\end{aligned}$ & $\begin{array}{r}11,26 \\
6,81\end{array}$ \\
\hline P 1000 & $\begin{array}{l}\text { (a) } \\
\text { (b) }\end{array}$ & $\begin{array}{l}5,181 \pm 0,113 \\
4,110 \pm 0,040\end{array}$ & $\begin{array}{l}0,532 \\
0,096\end{array}$ & $\begin{array}{l}5,282 \pm 0,051 \\
4,347 \pm 0,026\end{array}$ & $\begin{array}{l}0,481 \\
0,050\end{array}$ \\
\hline VERSE & $\begin{array}{l}\text { (a) } \\
\text { (b) }\end{array}$ & $\begin{array}{c}2,98 \pm 0,19 \\
-\end{array}$ & 1,965 & $\begin{array}{c}3,18 \pm 0,09 \\
-\end{array}$ & $\begin{array}{c}0,404 \\
-\end{array}$ \\
\hline NG/SIL.I & $\begin{array}{l}\text { (a) } \\
\text { (b) }\end{array}$ & $\begin{array}{c}12,82 \pm 0,60 \\
-\end{array}$ & $\begin{array}{l}3,519 \\
-\end{array}$ & $\begin{array}{c}13,16 \pm 0,33 \\
-\end{array}$ & $\begin{array}{c}3,396 \\
-\end{array}$ \\
\hline NG/SIL.II & $\begin{array}{l}\text { (a) } \\
\text { (b) }\end{array}$ & $\begin{array}{c}9,174 \pm 0,519 \\
-\end{array}$ & $\begin{array}{c}3,205 \\
-\end{array}$ & $\begin{array}{c}9,178 \pm 0,255 \\
-\end{array}$ & 2,801 \\
\hline $\mathrm{NG} / \mathrm{SIL}$ & $\begin{array}{l}\text { (a) } \\
\text { (b) }\end{array}$ & $12,88 \pm 2,23$ & $-\overline{2,475}$ & $\frac{-}{13,79 \pm 1,50}$ & $-\overline{2,376}$ \\
\hline $\mathrm{PG} / \mathrm{SIL}$ & $\begin{array}{l}\text { (a) } \\
\text { (b) }\end{array}$ & $57,39 \pm 0,48$ & $91, \overline{2}$ & $65,06 \pm 0,31$ & 69,5 \\
\hline
\end{tabular}


TABLEAU 5

Estimation de la vigueur hybride dans les essais de 1979 (a) et 1981 (b).

Estimation of hybrid vigor in the two trials, 1979 (a) and 1981 (b)

\begin{tabular}{|c|c|c|c|c|c|}
\hline \multirow{2}{*}{ Caractère } & & \multicolumn{2}{|c|}{ Vigueur hybride exprimée en fonction du : } & \multicolumn{2}{|c|}{ Meilleur des 2 parents } \\
\hline & & Brut & $\%$ & Brut & $\%$ \\
\hline DF & $\begin{array}{l}\text { (a) } \\
\text { (b) }\end{array}$ & $\begin{array}{l}-0,77^{*} \pm 0,32 \\
-1,30^{*} \pm 0,38\end{array}$ & $\begin{array}{l}6,4 \\
8,0\end{array}$ & $\begin{array}{l}0,63^{*} \pm 0,35 \\
2,03^{*} \pm 0,47\end{array}$ & $\begin{array}{r}-3.1 \\
-17,8\end{array}$ \\
\hline $\mathrm{CF}$ & $\begin{array}{l}\text { (a) } \\
\text { (b) }\end{array}$ & $\begin{array}{l}1,87^{*} \pm 0,24 \\
0,80^{*} \pm 0,16\end{array}$ & $\begin{array}{l}55,4 \\
24,3\end{array}$ & $\begin{array}{l}0,87^{*} \pm 0,28 \\
0,42^{*} \pm 0,19\end{array}$ & $\begin{array}{l}20,2 \\
11,3\end{array}$ \\
\hline RENDT & $\begin{array}{l}\text { (a) } \\
\text { (b) }\end{array}$ & $\begin{array}{l}7,11^{*} \pm 0,78 \\
6,68^{*} \pm 1,58\end{array}$ & $\begin{array}{l}23,5 \\
42,7\end{array}$ & $\begin{array}{l}3,52^{*} \pm 0,90 \\
3,70^{*} \pm 1,80\end{array}$ & $\begin{array}{l}12,0 \\
20,2\end{array}$ \\
\hline P 1000 & $\begin{array}{l}\text { (a) } \\
\text { (b) }\end{array}$ & $\begin{array}{l}0,101^{*} \pm 0,072 \\
0,237^{*} \pm 0,069\end{array}$ & $\begin{array}{l}2,0 \\
5,8\end{array}$ & $\begin{array}{r}-0,384^{*} \pm 0,088 \\
-0,054 \pm 0,082\end{array}$ & $\begin{array}{l}-6,7 \\
-1,2\end{array}$ \\
\hline VERSE & $\begin{array}{l}\text { (a) } \\
\text { (b) }\end{array}$ & $\begin{array}{c}0,20 \pm 0,18 \\
-\end{array}$ & $\begin{array}{l}6,8 \\
-\end{array}$ & $\begin{array}{c}0,98^{*} \pm 0,21 \\
-\end{array}$ & $\begin{array}{l}-44,5 \\
-\end{array}$ \\
\hline NG/SIL. I & $\begin{array}{l}\text { (a) } \\
\text { (b) }\end{array}$ & $\begin{array}{c}0,341 \pm 0,395 \\
-\end{array}$ & $\begin{array}{l}2,7 \\
-\end{array}$ & $\begin{array}{c}-0,925 \pm 0,491 \\
-\end{array}$ & $\begin{array}{l}-6,6 \\
-\end{array}$ \\
\hline NG/SIL. II & $\begin{array}{l}\text { (a) } \\
\text { (b) }\end{array}$ & $\begin{array}{c}0,0038 \pm 0,3616 \\
-\end{array}$ & $\stackrel{0,04}{-}$ & $\begin{array}{c}-1,51 \pm 0,557 \\
-\end{array}$ & $-14,1$ \\
\hline NG/SIL & $\begin{array}{l}\text { (a) } \\
\text { (b) }\end{array}$ & $0,91 \overline{1} \pm 0,818$ & $\overline{7,1}$ & $-0,494 \pm 0,972$ & $\begin{array}{l}- \\
-3,5\end{array}$ \\
\hline $\mathrm{PG} / \mathrm{SIL}$ & $\begin{array}{l}\text { (a) } \\
\text { (b) }\end{array}$ & $7,67 \pm 3,88$ & $\overline{13,4}$ & $1,72 \pm 4,61$ & - \\
\hline
\end{tabular}

* Significatif au seuil de $5 \%$.

* Significatif with $5 \%$ risk.

Si on se réfère au meilleur des 2 parents, la supériorité des hybrides n'est significative que pour le rendement et le couvert foliaire. Par contre, les hybrides, peut-être du fait de leur plus grande hauteur, sont nettement plus sensibles à la verse que le meilleur de leur parent.

Pour le rendement en grains, les estimations de la vigueur hybride (de l'ordre de 7 et $3,5 \mathrm{q} / \mathrm{ha}$ par rapport respectivement aux parents moyen et maximum) sont assez semblables en 1979 et 1981 . Or, en 1981, du fait des mauvaises conditions climatiques durant la phase de maturation, les moyennes des 2 populations ont été inférieures à celles de 1979 (tabl. 4); il en résulte que la vigueur hybride relative est nettement plus élevée en 1981 (43 p. 100 du parent moyen) qu'en 1979 (23 p. 100 du parent moyen).

Les conclusions précédentes ne concernent que les moyennes des 2 populations ; la précision des expériences ne permet pas en effet de comparer les estimations variétales individuelles. Cependant, 2 points doivent être soulignés ;

- dans les 2 essais, 30 p. 100 au moins des hybrides surpassent de plus de 10 p. 100 le rendement du meilleur cultivar "Jet neuf ";

- la vigueur toujours élevée des hybrides issus de croisements entre lignées européenne et asiatique suggère l'existence d'une bonne complémentation génique entre ces 2 types de lignées.

\section{Discussion}

Dans la population de lignées comme dans celle des hybrides, la distribution des caractères est proche de la normalité ; de plus, quel que soit le caractère, aucune discontinuité entre les 2 distributions n'est mise en évidence.

Les corrélations entre les résidus des caractères traités 2 à 2 ne sont pas significativement différentes et les matrices de covariances sont voisines chez les lignées et chez les hybrides. Bien que les estimations de covariances dépendent de la métrique utilisée, la similitude des variances résiduelles permet de penser que l'homogénéité des covariances correspond à une homogénéité des corrélations dans les 2 populations. Les relations entre caractères vis-à-vis de l'environnement ne semblent donc pas modifiées par l'état d'hétérozygotie. En 1979, des résultats similaires avaient déjà été mis en évidence pour l'ensemble des composantes du rendement des ramifications principales et secondaires (LEFORT-BUSON \& DATTÉE, 1982). De plus, l'homogénéité des estimations des corrélations dans les essais de 1979 et 1981 renforce la valeur de ces conclusions. Cet ensemble de résultats met en évidence une grande similitude de comportement des populations homozygotes et hétérozygotes.

Par ailleurs, les estimations de la variance génétique fluctuent selon les années. Toutefois, pour une année donnée, elles sont du même ordre de grandeur chez les lignées et chez les hybrides pour la majorité des caractères. Ce fait, qui pourrait traduire une analogie de l'expression génétique dans les 2 populations, sera plus amplement discuté dans le $2^{\mathrm{e}}$ article consacré à la décomposition de la variance génétique.

La comparaison des moyennes des 2 populations souligne une supériorité des hybrides sur les lignées pour tous les caractères à l'exception de la résistance à la verse. Si on se réfère à la meilleure des 2 lignées parentales, la vigueur hybride moyenne ne reste significative que pour le rendement et le couvert foliaire au 
début floraison. La supériorité moyenne des hybrides justifie donc la production de semences F1 ; toutefois, la vigueur hybride varie selon les conditions environnementales. Dans les conditions climatiques défavorables de 1981 , la supériorité relative des hybrides a été plus élevée qu'en 1979, bien qu'en moyenne la vigueur hybride ait été plus faible. Comme l'ont déjà suggéré LERNER (1954) et SCHWARTZ (1971), la diversité de leur stock génétique conférerait aux hybrides une plus grande homéostase. Orozco (1975) préconise d'ailleurs de prendre en compte le milieu dans toute estimation et interprétation de l'hétérosis.

Conformément aux études de GRAFFIUS (1960) sur le maïs et de DUARTE \& ADAMS (1972) sur le haricot, chez le colza la valeur hybride est plus importante pour des caractères «globaux » comme le rendement que pour des caractères "simples » comme les composantes. Le même résultat avait été établi lors de l'analyse de la vigueur hybride au cours du développement végétatif (BUSON, 1979) : la supériorité des hybrides était plus élevée pour le poids de matière sèche des parties aériennes que pour le nombre, la longueur et le poids des feuilles. L'homogénéité des distributions et des variances génétiques des 2 populations permet de penser que l'importance de l'hétérosis sur les caractères globaux est davantage liée à un cumul des supériorités observées pour les caractères élémentaires.

\section{CONCLUSION}

Chez le colza oléagineux d'hiver, les effets d'hétérosis chez les hybrides F1 issus de la population de lignées sélectionnées sont nets. L'hétérosis se manifeste plus ou moins fortement selon les caractères mesurés au cours du développement de la plante. Cependant, pour ces caractères, on observe une grande similitude de l'expression des effets respectivement génétiques et environnementaux (résiduels) chez les lignées et les hybrides. Pour les différents caractères, l'analogie des histogrammes de fréquence, d'une part, et des effets résiduels, d'autre part, chez les homozygotes et les hétérozygotes, suggère leur appartenance à des populations ne différant que par leurs valeurs moyennes. De plus, la similitude des variances génétiques est un argument en faveur d'une fixation possible de l'hétérosis chez les lignées à long terme.

A court et moyen terme, la production de semences hybrides se justifie puisque de très nombreux hybrides surpassent le cultivar «Jet Neuf» d'au moins 10 p. 100 . Il reste cependant nécessaire de disposer d'un système de stérilité mâle opérationnel, différents systèmes étant actuellement étudiés au laboratoire de Rennes.

Par ailleurs, la production de semences hybrides passe par un changement complet de la méthode de sélection employée jusqu'à maintenant. Or, la sélection pour la valeur hybride nécessite la connaissance de l'organisation de la variabilité génétique de la population ; l'étude de ce dernier point fera l'objet d'un $2^{\mathrm{e}}$ article.

Recu le 7 mai 1984. Accepté le 11 septembre 1984.

\section{REMERCIEMENTS}

Nous remercions très sincèrement l'ensemble des techniciens du Laboratoire Colza de la Station d'Amélioration des Plantes de Rennes-Le Rheu pour leur assistance tout au long de ce travail.

ANNEXE I

Estimations des variances résiduelles de chaque diallèle dans les essais de 1979, chez les hybrides Fl (i) et chez les lignées parentales (ii). Estimation of each diallel residual variance in the 1979 trial for the two populations, Fl hybrids (i) and selfed lines (ii).

\begin{tabular}{|c|c|c|c|c|c|c|c|c|c|}
\hline \multicolumn{2}{|c|}{ DIALLELE } & \multirow{2}{*}{$\begin{array}{c}\text { DDL } \\
14 \\
10\end{array}$} & \multirow{2}{*}{$\begin{array}{c}\mathrm{DF} \\
1,495 \\
1,453\end{array}$} & \multirow{2}{*}{$\begin{array}{c}\text { CF } \\
0,945 \\
1,173\end{array}$} & \multirow{2}{*}{$\begin{array}{c}\text { RENDT } \\
26,72 \\
25,02\end{array}$} & \multirow{2}{*}{$\begin{array}{l}\mathrm{P} 1000 \\
0,1392 \\
0,1639\end{array}$} & \multirow{2}{*}{$\begin{array}{c}\text { VERSE } \\
0,419 \\
0,497\end{array}$} & \multirow{2}{*}{$\begin{array}{c}\text { NB/SIL. RI } \\
\begin{array}{c}14,172 \\
4,339\end{array}\end{array}$} & \multirow{2}{*}{$\begin{array}{c}\text { NB/SIL. RII } \\
4,542 \\
3,268\end{array}$} \\
\hline 1 & $\begin{array}{r}\text { (i) } \\
\text { (ii) }\end{array}$ & & & & & & & & \\
\hline 2 & $\begin{array}{r}\text { (i) } \\
\text { (ii) }\end{array}$ & $\begin{array}{r}17 \\
7\end{array}$ & $\begin{array}{l}1,261 \\
2,533\end{array}$ & $\begin{array}{l}1,080 \\
1,542\end{array}$ & $\begin{array}{l}39,15 \\
39,46\end{array}$ & $\begin{array}{l}0,1743 \\
0,3661\end{array}$ & $\begin{array}{l}0,867 \\
0,804\end{array}$ & $\begin{array}{r}6,313 \\
10,082\end{array}$ & $\begin{array}{l}3,515 \\
5,960\end{array}$ \\
\hline 3 & $\begin{array}{r}\text { (i) } \\
\text { (ii) }\end{array}$ & $\begin{array}{l}16 \\
10\end{array}$ & $\begin{array}{l}2,020 \\
1,998\end{array}$ & $\begin{array}{l}0,810 \\
0,866\end{array}$ & $\begin{array}{l}28,66 \\
28,03\end{array}$ & $\begin{array}{l}0,1507 \\
0,1527\end{array}$ & $\begin{array}{l}0,453 \\
0,583\end{array}$ & $\begin{array}{l}6,285 \\
5,850\end{array}$ & $\begin{array}{l}4,677 \\
5,080\end{array}$ \\
\hline 4 & $\begin{array}{r}\text { (i) } \\
\text { (ii) }\end{array}$ & $\begin{array}{r}16 \\
9\end{array}$ & $\begin{array}{l}1,202 \\
1,385\end{array}$ & $\begin{array}{l}0,673 \\
1,005\end{array}$ & $\begin{array}{l}23,18 \\
34,51\end{array}$ & $\begin{array}{l}0,2102 \\
0,2670\end{array}$ & $\begin{array}{l}0,598 \\
0,721\end{array}$ & $\begin{array}{l}8,485 \\
5,388\end{array}$ & $\begin{array}{l}5,514 \\
6,006\end{array}$ \\
\hline 5 & $\begin{array}{r}\text { (i) } \\
\text { (ii) }\end{array}$ & $\begin{array}{r}17 \\
7\end{array}$ & $\begin{array}{l}1,014 \\
1,260\end{array}$ & $\begin{array}{l}0,924 \\
1,644\end{array}$ & $\begin{array}{l}15,05 \\
30,14\end{array}$ & $\begin{array}{l}0,2234 \\
0,2727\end{array}$ & $\begin{array}{l}0,569 \\
0,807\end{array}$ & $\begin{array}{l}6,446 \\
6,549\end{array}$ & $\begin{array}{l}4,241 \\
6,154\end{array}$ \\
\hline 6 & $\begin{array}{r}\text { (i) } \\
\text { (ii) }\end{array}$ & $\begin{array}{r}19 \\
7\end{array}$ & $\begin{array}{l}1,413 \\
1,312\end{array}$ & $\begin{array}{l}1,417 \\
1,031\end{array}$ & $\begin{array}{l}12,52 \\
18,37\end{array}$ & $\begin{array}{l}0,1495 \\
0,1494\end{array}$ & $\begin{array}{l}0,336 \\
0,424\end{array}$ & $\begin{array}{l}7,044 \\
8,001\end{array}$ & $\begin{array}{l}6,798 \\
3,258\end{array}$ \\
\hline 7 & $\begin{array}{r}\text { (i) } \\
\text { (ii) }\end{array}$ & $\begin{array}{r}17 \\
7\end{array}$ & $\begin{array}{l}1,500 \\
2,176\end{array}$ & $\begin{array}{l}0,825 \\
1,197\end{array}$ & $\begin{array}{l}35,21 \\
28,40\end{array}$ & $\begin{array}{l}0,2334 \\
0,2264\end{array}$ & $\begin{array}{l}0,847 \\
0,795\end{array}$ & $\begin{array}{l}5,623 \\
5,412\end{array}$ & $\begin{array}{l}4,439 \\
3,344\end{array}$ \\
\hline GLOBAL & $\begin{array}{l}\text { (i) } \\
\text { (ii) }\end{array}$ & $\begin{array}{r}116 \\
57\end{array}$ & $\begin{array}{l}1,406 \\
1,718\end{array}$ & $\begin{array}{l}0,956 \\
1,181\end{array}$ & $\begin{array}{l}25,53 \\
29,05\end{array}$ & $\begin{array}{l}0,1838 \\
0,2223\end{array}$ & $\begin{array}{l}0,591 \\
0,651\end{array}$ & $\begin{array}{l}7,689 \\
6,328\end{array}$ & $\begin{array}{l}4,508 \\
4,711\end{array}$ \\
\hline
\end{tabular}


ANNEXE II

Estimations des variances résiduelles de chaque diallèle dans les essais de 1981, chez les hybrides FI (i) et chez les lignées parentales (ii). Estimation of each diallel residual variance in the 1981 trial for the two populations, F1 hybrids (i) and selfed lines (ii).

\begin{tabular}{|c|c|c|c|c|c|c|c|c|}
\hline DIALLELE & & DDL & $\mathrm{DF}$ & $\mathrm{CF}$ & RENDT & P 1000 & NB/SIL & $\mathrm{PG} / \mathrm{SIL}$ \\
\hline 1 & $\begin{array}{l}\text { (i) } \\
\text { (ii) }\end{array}$ & $\begin{array}{l}26 \\
15\end{array}$ & $\begin{array}{l}0,758 \\
1,200\end{array}$ & $\begin{array}{l}0,2557 \\
0,2344\end{array}$ & $\begin{array}{l}27,40 \\
26,94\end{array}$ & $\begin{array}{l}0,0431 \\
0,0418\end{array}$ & $\begin{array}{l}5,252 \\
7,460\end{array}$ & $\begin{array}{l}139,2 \\
134,4\end{array}$ \\
\hline 2 & $\begin{array}{l}\text { (i) } \\
\text { (ii) }\end{array}$ & $\begin{array}{l}27 \\
15\end{array}$ & $\begin{array}{l}0,814 \\
2,942\end{array}$ & $\begin{array}{l}0,2671 \\
0,3492\end{array}$ & $\begin{array}{l}33,14 \\
19,46\end{array}$ & $\begin{array}{l}0,0282 \\
0,0376\end{array}$ & $\begin{array}{l}6,678 \\
6,475\end{array}$ & $\begin{array}{l}137,1 \\
133,7\end{array}$ \\
\hline 3 & $\begin{array}{r}\text { (i) } \\
\text { (ii) }\end{array}$ & $\begin{array}{l}27 \\
10\end{array}$ & $\begin{array}{l}0,733 \\
1,418\end{array}$ & $\begin{array}{l}0,3220 \\
0,3239\end{array}$ & $\begin{array}{l}25,95 \\
17,20\end{array}$ & $\begin{array}{l}0,0442 \\
0,0566\end{array}$ & $\begin{array}{l}6,516 \\
7,762\end{array}$ & $\begin{array}{l}111,2 \\
165,1\end{array}$ \\
\hline 4 & $\begin{array}{l}\text { (i) } \\
\text { (ii) }\end{array}$ & $\begin{array}{l}24 \\
13\end{array}$ & $\begin{array}{l}1,409 \\
1,339\end{array}$ & $\begin{array}{l}0,2089 \\
0,3209\end{array}$ & $\begin{array}{l}31,19 \\
22,19\end{array}$ & $\begin{array}{l}0,0517 \\
0,0520\end{array}$ & $\begin{array}{l}6,546 \\
7,649\end{array}$ & $\begin{array}{l}141,8 \\
164,5\end{array}$ \\
\hline 5 & $\begin{array}{r}\text { (i) } \\
\text { (ii) }\end{array}$ & $\begin{array}{c}27 \\
15\end{array}$ & $\begin{array}{l}1,178 \\
1,110\end{array}$ & $\begin{array}{l}0,2435 \\
0,3056\end{array}$ & $\begin{array}{l}21,91 \\
19,33\end{array}$ & $\begin{array}{l}0,0510 \\
0,0489\end{array}$ & $\begin{array}{l}5,357 \\
7,222\end{array}$ & $\begin{array}{l}151,0 \\
144,0\end{array}$ \\
\hline 6 & $\begin{array}{l}\text { (i) } \\
\text { (ii) }\end{array}$ & $\begin{array}{l}23 \\
11\end{array}$ & $\begin{array}{l}1,482 \\
2,101\end{array}$ & $\begin{array}{l}0,2737 \\
0,2079\end{array}$ & $\begin{array}{l}21,49 \\
24,12\end{array}$ & $\begin{array}{l}0,0291 \\
0,0563\end{array}$ & $\begin{array}{l}5,071 \\
5,590\end{array}$ & $\begin{array}{l}126,2 \\
152,6\end{array}$ \\
\hline GLOBAL & $\begin{array}{l}\text { (i) } \\
\text { (ii) }\end{array}$ & $\begin{array}{r}154 \\
79\end{array}$ & $\begin{array}{l}1,051 \\
1,685\end{array}$ & $\begin{array}{l}0,2613 \\
0,2903\end{array}$ & $\begin{array}{l}26,89 \\
21,54\end{array}$ & $\begin{array}{l}0,0413 \\
0,0489\end{array}$ & $\begin{array}{l}5,924 \\
7,026\end{array}$ & $\begin{array}{l}134,4 \\
149,1\end{array}$ \\
\hline
\end{tabular}

ANNEXE III

Histogrammes des moyennes variétales pour le rendement

et quelques composantes.

Histograms of varietal means for seed yield and some of its components.
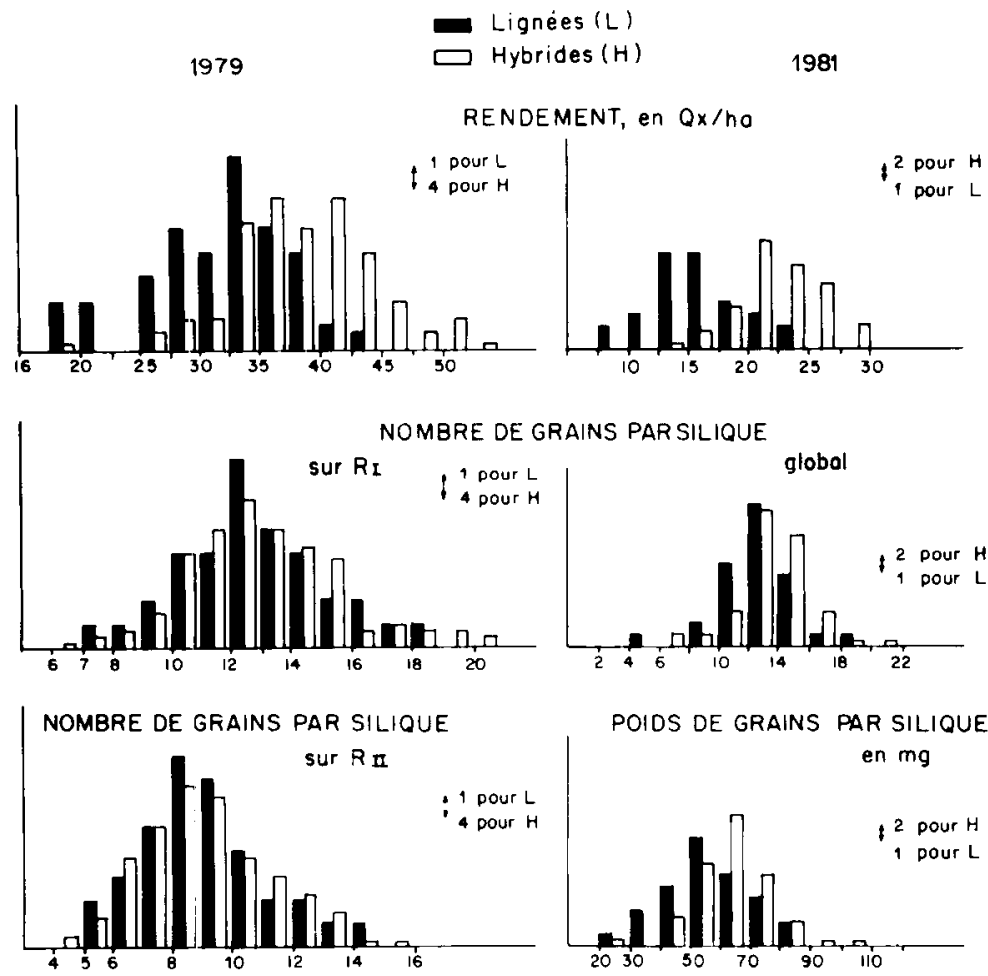

POIDS DE MILLE GRAINS, en $g$.

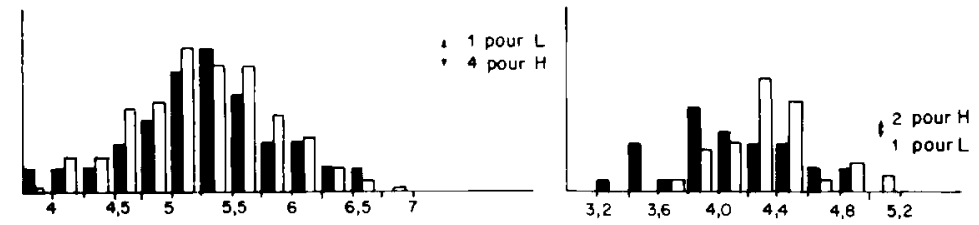




\section{ANNEXE IV}

Histogrammes des moyennes variétales pour la précocité de floraison, le couvert foliaire et la sensibilité à la verse.

Histograms of varietal means for the earliness of flowering period, the leaf canopy and the susceptibility to lodging.

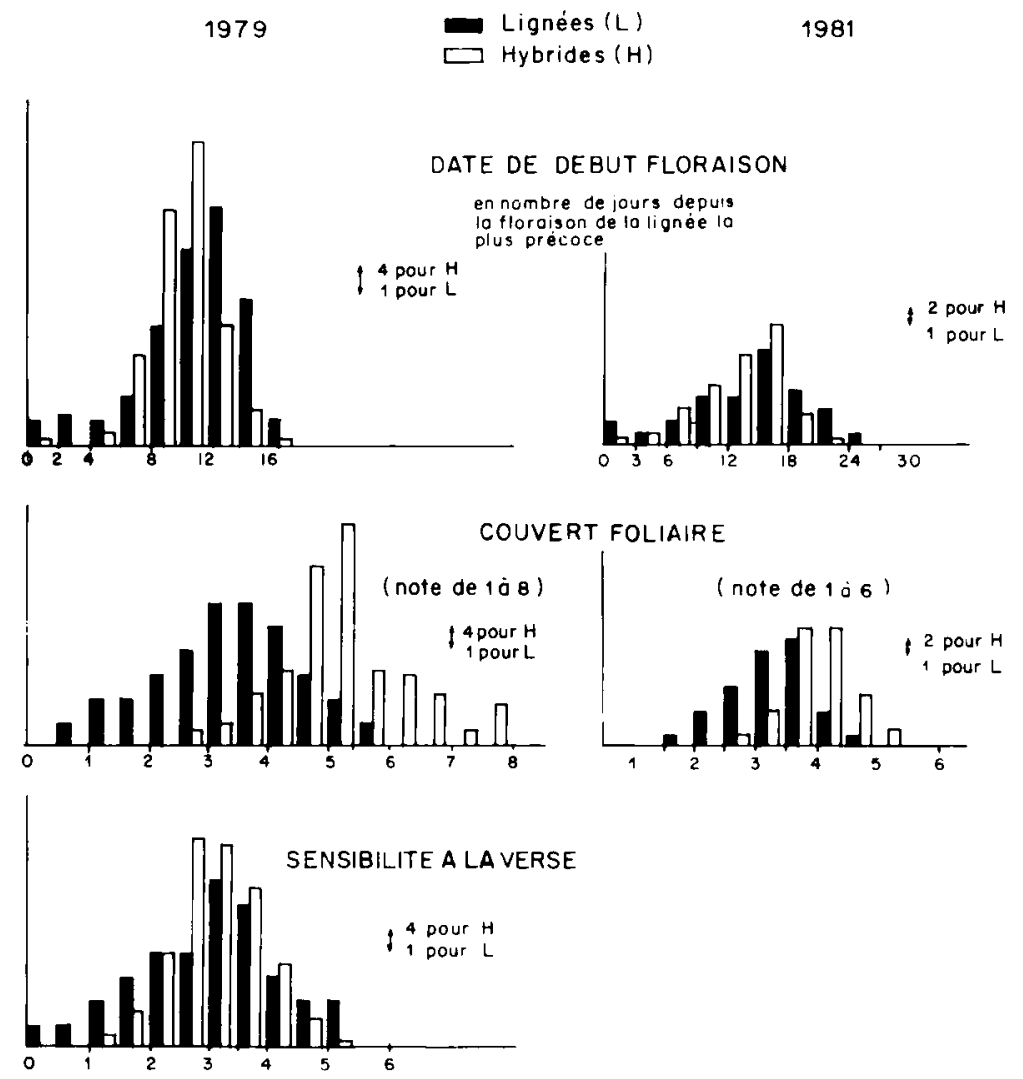

RÉFÉRENCES BIBLIOGRAPHIQUES

Anderson T. W., 1957. Testing hypotheses of equality of covariance matrices and equality of mean vectors and covariances matrices, 247-271. In "An introduction to multivariate analysis". John Wiley Son, New York, $372 \mathrm{p}$.

Buson M., 1979. Hétérosis et paramètres génétiques de quelques caractères agronomiques chez le colza oléagineux (Brassica napus L.). Thèse Doct. $3^{\text {e }}$ cycle, Paris VI, $78 \mathrm{p}$.

Campbel D. C., Kondra Z. P., 1978. A genetic study of growth characters and yield characters in oilseed rape. Euphytica, 27 (1), 177-183.

Duarte R. A., Adams M. W., 1972. A path coefficient analysis of some yield component interrelations in field beans (Phaseolus vulgaris L.). Crop Sci., 22, 579-582.

Graffius J. E., 1960. Does overdominance exist for yield in corn ?, Agron. J., 52, 361.

Lefort-Buson M., 1981. Competition effects between rows of neighbouring plots of winter rapeseed (Brassica napus L.). Cruciferae Newsletter, 6, 26-27.

Lefort-Buson, M. Dattée Y., 1982. Genetic study of some agronomic characters in winter oilseed rape (Brassica napus L.) I. Heterosis. Agronomie, 2 (4), 315-322.

Lerner I. M., 1954. Genetics of homeostasis. Oliver \& Boyd, Edinburgh, $134 \mathrm{p}$.

Orozco F., 1975. Heterosis and genotype-environment interaction : theoretical and experimental aspects. Bull. techn. Dept. Gen. anim., $24,43-52$.
Rousselle P., 1982. Premiers résultats d'un programme d'introduction de l'andro-stérilité "ogura " du radis chez le colza. Agronomie, 2 (9), 859-864.

Rousselle P., Renard M., 1982. Intérêt du cultivar "Bronowski " pour l'obtention de plantes mâles-stériles cytoplasmiques chez le colza. Agronomie, 2 (10), 951-956.

Schuster W., 1969. Vergleich von zwei Zuchtverfahren in der Erhaltungszüchtung bei Winterraps. Z. Pflanzenzücht, 62, 47-62.

Schuster W., Michael J., 1976. Untersuchungen über Inzuchtdepressionen und Heterosiseffekte bei Raps (Brassica napus oleifera). Z. Pflanzenzücht., 77, 56-66.

Schwartz D., 1971. Genetic control of alcohol dehydrogenase. A competition model for regulation of gene action. Genetics, 67, 411-425.

Shelkudenko V. G., 1968. First results of evaluation of intervarietal hybrids of winter rape for heterosis. Sb. nauchno-issled. Rab. maslichn. kul't., 9-14 (in Russian).

Shelkudenko V. G., 1972. Early diagnosis of heterosis in rape hybrids. Sel. Semenovod., 37 (1), 17 (in Russian).

Shiga T., 1976. Studies on heterosis breeding using cytoplasmic male sterility in rape. Bull. Nat. Inst. Agric. Sci., 27, 1-101.

Thompson K. F., 1972. Cytoplasmic male sterility in oil-seed rape. Heredity, 29, 253-257. 